\title{
Interpolative Approach for Solving the Anderson Impurity Model
}

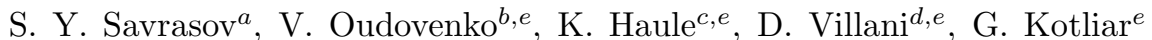 \\ ${ }^{a}$ Department of Physics, New Jersey Institute of Technology, Newark, NJ 07102, USA \\ ${ }^{b}$ Laboratory for Theoretical Physics, Joint Institute for Nuclear Research, 141980 Dubna, Russia \\ ${ }^{c}$ Jožef Stefan Institute, SI-1000 Ljubljana, Slovenia \\ ${ }^{d}$ JPMorgan Chase Bank, 270 Park Avenue, New York, NY 10017 and \\ ${ }^{e}$ Department of Physics and Center for Material Theory, \\ Rutgers University, Piscataway, NJ 08854, USA
}

(Dated: November 21, 2018)

\begin{abstract}
A rational representation for the self-energy is explored to interpolate the solution of the Anderson impurity model in general orbitally degenerate case. Several constrains such as the Friedel's sum rule, positions of the Hubbard bands as well as the value of quasiparticle residue are used to establish the equations for the coefficients of the interpolation. We employ two fast techniques, the slaveboson mean-field and the Hubbard I approximations to determine the functional dependence of the coefficients on doping, degeneracy and the strength of the interaction. The obtained spectral functions and self-energies are in good agreement with the results of numerically exact quantum Monte Carlo method.

PACS numbers: 71.10.-w, 71.27.+a, 71.30.+h
\end{abstract}

\section{INTRODUCTION}

There has been recent progress in understanding physics of strongly correlated electronic systems and their electronic structure near a localization-delocalization transition through the development of dynamical meanfield theory (DMFT) $\frac{1}{\underline{1}}$. Merging this computationally tractable many-body technique with realistic localdensity-approximation (LDA) $)^{2}$ based electronic structure calculations of strongly correlated solids is promising due to its simplicity and correctness in both band and atomic limits. At present, much effort is being made in this direction including the developments of a LDA+DMFT method ${ }^{3}$, LDA ++ approach $^{4}$, combined GW and DMFT theory $\underline{5}^{\underline{5}}$, spectral density functional theory ${ }^{\underline{6}}$ as well as applications to various systems such as $\mathrm{La}_{1-x} \mathrm{Sr}_{x} \mathrm{TiO}_{3}{ }^{7}, \mathrm{~V}_{2} \mathrm{O}_{3}{ }^{8}, \mathrm{Fe}$ and $\mathrm{Ni}^{9}, \mathrm{Ce}^{10}, \mathrm{Pu}^{11.12}$, transition metal oxides $\frac{13}{3}$, and many others. For a review, see Ref. 14.

Such ab initio DMFT based self-consistent electronic structure algorithms should be able to explore all space of parameters where neither dopings nor even degeneracy itself is kept fixed as different states may appear close to the Fermi level during iterations towards self-consistency. This is crucial if one would like to calculate properties of realistic solid state system where bandwidth and the strength of the interaction is not known at the beginning. It is very different from the ideology of model Hamiltonians where the input set of parameters defines the regime of correlations, and the corresponding many-body techniques may be applied afterwards. Realistic DMFT simulations of material properties require fast scans of the entire parameter space to determine the interaction for a given doping, degeneracy and bandwidth via the solution of the general multiorbital Anderson impurity model $(\mathrm{AIM}) \frac{15}{15}$. Unfortunately, present approaches based on either non-crossing approximation (NCA) or iterative per- turbation theory (IPT) are unable to provide the solution to that problem due to a limited number of regimes where these methods can be applied ${ }^{\underline{1}}$. The quantum Monte Carlo (QMC) technique ${ }^{1.16}$ is very accurate and can cope with multiorbital situation but not with multiplet interactions. Also its applicability so far has been limited either to a small number of orbitals or to unphysically large temperatures due to its computational cost. Recently some progress has been achieved using impurity solvers that improve upon the NCA approximation:17,18.19, but it has not been possible to retrieve Fermi liquid behavior at very low temperatures with these methods in the orbitally degenerate case.

As universal impurity solvers have not yet being designed in the past we need to explore other possibilities, and this paper proposes interpolative approach for the self-energy in general multiorbital situation. We stress that this is not an attempt to develop an alternative method for solving the impurity problem, but followup of the ideology of LDA theory where approximations were designed by analytical fits ${ }^{20}$ to the quantum Monte Carlo simulations for homogeneous electron gas 21 . Numerically very expensive QMC calculations for the impurity model display smooth self-energies at imaginary frequencies for a wide range of interactions and dopings, and it is therefore tempting to design such an interpolation. We also keep in mind that for many applications a high precision in reproducing the self-energies may not be required. One of such applications is, for example, the calculation of the total energy ${ }^{10.11,12.13}$ which, as well known from LDA based experience, may not be so sensitive to the details of the one-electron spectra. As a result, we expect that even crude evaluations of the self-energy shapes on imaginary axis may be sufficient for solving many realistic total energy problems, some of which have appeared already ${ }^{11.12 .13}$. Another point is a computational efficiency and numerical stability. Bringing full self-consistent loops with respect to charge densities ${ }^{11}$ 
and other spectral functions require many iterations towards the convergency which may not need too accurate frequency resolutions at every step. However, the procedure which solves the impurity model should smoothly connect various regions of the parameter space. This is a crucial point if one would like to have a numerically stable algorithm and our new interpolational approach ideally solves this problem.

In the calculations of properties such as the low-energy spectroscopy and especially transport more delicate distribution of spectral weight is taken place at low energies, and the imaginary part of the analytically continued self-energy needs to be computed with a greater precision. Here we expect that our obtained spectral functions should be used with care. Also, in a few well distinct regimes, such, e.g., as very near the Mott transition, the behavior maybe much more complicated and more difficult to interpolate. For the cases mentioned above extensions of the interpolative methods should be implemented and its beyond the scope of the present work.

We can achieve a fast interpolative algorithm for the self-energy by utilizing a rational representation. The coefficients in this interpolation can be found by forcing the self-energy to obey several limits and constrains. For example, if infinite frequency (Hartree-Fock) limit, positions of the Hubbard bands, low-frequency mass renormalization $z$, mean number of particles $\bar{n}$ as well as the value of the self-energy at zero frequency $\Sigma(0)$ are known from independent calculation, the set of interpolating coefficients is well defined. In this work, we explore the slave-boson mean-field (SBMF) approach ${ }^{22.23 .24 .25}$ and the Hubbard I approximation ${ }^{26}$ to determine the functional dependence of these coefficients upon doping, degeneracy and the strength of the interaction $U$. We verify all trends produced by this interpolative procedure in the regimes of weak, intermediate and strong interactions and at various dopings conditions. These trends are compared with known analytical limits as well as against calculations using the quantum Monte Carlo method. Also, compared with QMC are self-energies and spectral functions on both imaginary and real axes for selective values of dopings. They indicate that the SBMF approach can predict such parameters of interpolation as $\bar{n}, \Sigma(0)$ and $z$ with a good accuracy while the Hubbard I method fails in a number of regimes. However, the functional form of the atomic Green function which appears within Hubbard I can be used to determine positions of atomic satellites which helps to impose additional constraints on our procedure.

Giving an extraordinary computational speed of this approach we generally find a very satisfactory accuracy in comparisons with the numerically more accurate QMC calculations. If an increased accuracy is desired our method can be naturally extended by imposing more constraints and by implementing more refined impurity solvers other than the ones explored in this work.

The paper is organized as follows. In Section II we discuss rational interpolation for the self-energy and list the constraints. In Section III we discuss methods for solving Anderson impurity model such as the slave-boson mean field and the Hubbard I approximations which can be used to find these constraints. A brief survey of the QMC method used to benchmark our algorithm is also given. We present numerical comparisons of SBMF and Hubbard I techniques against the QMC simulations for such quantities as quasiparticle residue and multiple occupancies. In Section IV we report the results of the interpolative method and compare the obtained spectral functions with the QMC. In Section V we discuss possible improvements of the algorithm. Section VI is the conclusion.

\section{INTERPOLATIVE APPROACH}

To be specific, we concentrate on the Anderson impurity Hamiltonian

$$
\begin{aligned}
H & =\epsilon_{f} \sum_{\alpha=1}^{N} f_{\alpha}^{+} f_{\alpha}+\frac{1}{2} U \sum_{\alpha \neq \beta}^{N} n_{\alpha}^{f} n_{\beta}^{f}+\sum_{\mathbf{k} \alpha} E_{\mathbf{k} \alpha} c_{\mathbf{k} \alpha}^{+} c_{\mathbf{k} \alpha} \\
& +\sum_{\mathbf{k} \alpha}\left[V_{\alpha}^{*}(\mathbf{k}) f_{\alpha}^{+} c_{\mathbf{k} \alpha}+V_{\alpha}(\mathbf{k}) c_{\mathbf{k} \alpha}^{+} f_{\alpha}\right]
\end{aligned}
$$

describing the interaction of the impurity level $\epsilon_{f}$ with bands of conduction electrons $E_{\mathbf{k} \alpha}$ via hybridization $V_{\alpha}(\mathbf{k}) . \quad U$ is the Coulomb repulsion between different orbitals in the $f$-band. Inspired by the success of the iterative perturbation theory ${ }^{\underline{1}}$, in order to solve the Anderson impurity model in general multiorbital case, we use a rational interpolative formula for the self-energy. This can be encoded into a form

$$
\Sigma(\omega)=\frac{\sum_{m=0}^{M} a_{m} \omega^{m}}{\sum_{m=0}^{M} b_{m} \omega^{m}}=\Sigma(\infty) \frac{\prod_{m=1}^{M}\left[\omega-Z_{m}^{(\Sigma)}\right]}{\prod_{m=1}^{M}\left[\omega-P_{m}^{(\Sigma)}\right]},
$$

The coefficients $a_{m}, b_{m}$, or, alternatively, the poles $P_{m}^{(\Sigma)}$, zeroes $Z_{m}^{(\Sigma)}$ and $\Sigma(\infty)$ in this equation are to be determined. The form (2) can be also viewed as a continuous fraction expansion but continuous fraction representation will not be necessary for the description of the method.

Our basic assumption is that only a well distinct set of poles in the rational representation (2) is necessary to reproduce an overall frequency dependence of the selfenergy. Extensive experience gained from solving Hubbard and periodic Anderson model within DMFT at various ratios of the on-site Coulomb interaction $U$ to the bandwidth $W$ shows the appearance of lower and upper Hubbard bands as well as renormalized quasiparticle peak in the spectrum of one-electron excitations ${ }^{1}$.

It is clear that the Hubbard bands are damped atomic excitations and to the lowest order approximation appear as the positions of the poles of the atomic Green function. In the $S U(N)$ symmetry case which is described by the 
Hamiltonian (1), these energies are numerated by the number of electrons occupying impurity level, i.e. $E_{n}=$ $\epsilon_{f} n+\frac{1}{2} U n(n-1)$, and the atomic Green function takes a simple functional form

$$
G_{a t}(\omega)=\sum_{n=0}^{N-1} \frac{C_{n}^{N-1}\left(X_{n}+X_{n+1}\right)}{\omega+\mu-E_{n+1}+E_{n}}
$$

where $X_{n}$ are the probabilities to find an atom in configuration with $n$ electrons while combinatorial factor $C_{n}^{N-1}=\frac{(N-1) !}{n !(N-n-1) !}$ arrives due to equivalence of all states with $n$ electrons in $S U(N)$.

We can represent the atomic Green function (3) using the rational representation (2), i.e.

$$
G_{a t}(\omega)=\frac{\prod_{n=1}^{N-1}\left[\omega-Z_{n}^{(G)}\right]}{\prod_{n=1}^{N}\left[\omega-P_{n}^{(G)}\right]}
$$

where $P_{n}^{(G)}$ are all $N$ atomic poles, while $Z_{n}^{(G)}$ denote $N-$ 1 zeroes with $N$ being the total degeneracy. The centers of the Hubbard bands are thus located at the atomic excitations $P_{n}^{(G)}=E_{n}-E_{n-1}-\mu=\epsilon_{f}-\mu-U(n-1)$. Using standard expression for the atomic Green function $G_{a t}(\omega)=1 /\left[\omega+\mu-\epsilon_{f}-\Sigma_{a t}(\omega)\right]$, we arrive to a desired representation for the atomic self-energy

$$
\Sigma^{(a t)}(\omega)=\omega+\mu-\epsilon_{f}-\frac{\prod_{n=1}^{N}\left[\omega-P_{n}^{(G)}\right]}{\prod_{n=1}^{N-1}\left[\omega-Z_{n}^{(G)}\right]} .
$$

Using this functional form for finite $\Delta(\omega)$ modifies the positions of poles and zeroes via recalculating probabilities $X_{n}$ which is equivalent to the famous Hubbard I approximation (discussed in more detail in the next Section).

We now concentrate on the description of the quasiparticle peak which is present in metallic state of the system. For this an extra pole and zero have to be added in Eq (5). To see this, let us consider the Hubbard model for the $S U(N)$ case where the local Green function can be written by the following Hilbert transform $G_{f}(\omega)=H\left[\omega+\mu-\epsilon_{f}-\Sigma(\omega)\right]$. If self-energy lifetime effects are ignored, the local spectral function becomes $N_{f}(\omega)=D\left[\omega+\mu-\epsilon_{f}-\Sigma(\omega)\right]$ where $D$ is the non-interacting density of states. The peaks of the spectral functions thus appear as zeroes in Eq. (5) and in order to add the quasiparticle peak, one needs to add one extra zero (denoted hereafter as $X$ ) to the numerator in Eq. (5). To make the self-energy finite at $\omega \rightarrow \infty$ one has to also add one more pole (denoted hereafter as $P_{1}^{(\Sigma)}$ ) which should appear in the denominator or Eq. (5). Furthermore, frequently the Hartree Fock value for the self-energy can be computed separately and it is desirable to have a parameter in the functional form (5) which will allow us to fix $\Sigma(\infty)$. An obvious candidate to be changed is that self-energy pole in (5) which is closest to $\omega$ equal zero. Let us denote this parameter as $P_{2}^{(\Sigma)}$ and rewrite the denominator of (5) as $\left(\omega-P_{1}^{(\Sigma)}\right)\left(\omega-P_{2}^{(\Sigma)}\right) \prod_{n=1}^{N-2}\left[\omega-Z_{n}^{(G)}\right]$ where the product is now extended over all zeroes of the atomic Green functions except the one closest to zero and two extra poles $P_{1}^{(\Sigma)}$ and $P_{2}^{(\Sigma)}$ can control the width of the quasiparticle peak and $\Sigma(\infty)$. Thus, we arrive to the functional form for the self-energy

$$
\Sigma(\omega)=\omega+\mu-\epsilon_{f}-\frac{(\omega-X) \prod_{n=1}^{N}\left[\omega-P_{n}^{(G)}\right]}{\left(\omega-P_{1}^{(\Sigma)}\right)\left(\omega-P_{2}^{(\Sigma)}\right) \prod_{n=1}^{N-2}\left[\omega-Z_{n}^{(G)}\right]}
$$

We are now ready to list all constrains of our interpolative scheme. To fix the unknown coefficients $X, P_{1}^{(\Sigma)}$, $P_{2}^{(\Sigma)}, P_{n}^{(G)}, Z_{n}^{(G)}$ in Eq. (6) and to write down the linear set of equations for the coefficients $a_{m}, b_{m}$ in Eq. (2). we use the following set of conditions.

a) Hartree Fock value $\Sigma(\infty)$. In the limit $\omega \rightarrow \infty$ the self-energy takes its Hartree-Fock form

$$
\Sigma(\infty)=U(N-1)\langle n\rangle .
$$

Mean level occupancy $\langle n\rangle$ is defined as a sum over all
Matsubara frequencies for the Green's function, i.e.

$$
\langle n\rangle=T \sum_{i \omega} G_{f}(i \omega) e^{i \omega 0^{+}},
$$

where

$$
G_{f}(\omega)=\frac{1}{\omega+\mu-\epsilon_{f}-\Delta(\omega)-\Sigma(\omega)},
$$

defines the impurity Green function and $\Delta(\omega)$ is the hybridization function. 
b) Zero-frequency value $\Sigma(0)$. The so called Friedel sum rule establishes the relation between the total density and the real part of the self-energy at zero frequency

$$
\begin{aligned}
\langle n\rangle & =\frac{1}{2}+\frac{1}{\pi} \operatorname{arctg}\left(\frac{\epsilon_{f}+\Re \Sigma\left(i 0^{+}\right)+\Re \Delta\left(i 0^{+}\right)}{\Im \Delta\left(i 0^{+}\right)}\right) \\
& +\int_{-i \infty}^{+i \infty} \frac{d z}{2 \pi i} G_{f}(z) \frac{\partial \Delta(z)}{\partial z} e^{z 0^{+}} .
\end{aligned}
$$

c) Quasiparticle mass renormalization value $\partial \Re \Sigma /\left.\partial \omega\right|_{\omega=0}$. The slope of the self-energy at zero frequency controls the quasiparticle residue, $z$ using the following relationship

$$
\left.\frac{\partial \Re \Sigma}{\partial \omega}\right|_{\omega=0}=1-z^{-1} .
$$

Formally, constraints $(b)$ and $(c)$ hold for zero temperature only but we expect no significant deviations in many regions of parameters as long as we stay at low enough temperatures. The behavior may be more elaborated in the vicinity of Mott transition 27 .

d) Positions of Hubbard bands.

As we discussed, in order that the self-energy obeys the atomic limit and places the centers of the Hubbard bands at the positions of the atomic excitations, we demand that

$$
P_{n}^{(G)}+\mu-\epsilon_{f}=\Sigma\left(P_{n}^{(G)}\right)
$$

This condition fixes almost all self-energy zeroes $Z_{m}^{(\Sigma)}$ in Eq.(2) to the poles $P_{n}^{(G)}$. However, it alone does not ensure that the weight is correctly distributed among the Hubbard bands and that the very distant Hubbard bands disappear. For this to occur, distant poles of Green function have to be canceled out by nearby zeroes of the
Green function. It is clear that each pole $P_{n}^{(G)}$ far from the Fermi level has to be accompanied by a nearby zero $Z_{n}^{(G)}$ in order the weight of the pole be small. Thus, the self-energy has poles at the position of Green's function zeroes which can be encoded into the constrain

$$
\left[\Sigma\left(Z_{n}^{(G)}\right)\right]^{-1}=0
$$

We want to keep this property of the self-energy for finite $\Delta(\omega)$ and thus demand that self-energy diverges (when lifetime effects are kept, it only reaches a local maximum) at the zeros of the functional form (3) of $G_{a t}(\omega)$. Note that the relationship (13) holds (approximately) for frequency $\omega$ larger than the renormalized bandwidth $z W$. Therefore the information about one $Z_{n}^{(G)}$ which lies close to $\omega=0$ is omitted and replaced by the information about $\Sigma(\infty), \Sigma(0)$ and $z$ as it is done by separating $P_{1}^{(\Sigma)}$ and $P_{2}^{(\Sigma)}$ in the denominator of Eq. (6).

We can now write down a set of linear equations for all unknown coefficients in the expression (2). There is total $2 M+2$ of parameters $a_{m}$ and $b_{m}, m=0, M$, where we can always set $b_{0}=1$. The conditions $a$ ),b),c) give

$$
\begin{aligned}
a_{0} & =\Sigma(0), \\
b_{0} & =1 \\
a_{1}-\Sigma(0) b_{1} & =1-z^{-1}, \\
a_{M}-b_{M} \Sigma(\infty) & =0 .
\end{aligned}
$$

According to condition $d$ ) we can use all $N$ poles $P_{n}^{(G)}$ and $N-2$ zeroes $Z_{n}^{(G)}$. The zero $Z_{n}^{(G)}$ closest to $\omega=0$ is dropped out. This brings additional $2 N-2$ equations for the coefficients and makes $M=N$ as the degree of the rational interpolation which are written below

$$
\begin{aligned}
\sum_{m=0}^{N} a_{m}\left[P_{n}^{(G)}\right]^{m}-\left(P_{n}^{(G)}+\mu-\epsilon_{f}\right) & \sum_{m=0}^{N} b_{m}\left[P_{n}^{(G)}\right]^{m}=0 \text { for } n=1 \ldots N \\
\sum_{m=0}^{N} b_{m}\left[Z_{n}^{(G)}\right]^{m} & =0 \text { for } n=1 \ldots N-2 .
\end{aligned}
$$

Note that while $M$ may be rather large, the actual number of poles contributing to the self-energy behavior is indeed very small. We can directly see this from Eq. (5) which uses all $N$ poles $P_{n}^{(G)}$ fulfilling Eq. (12) and uses $N-2$ zeroes $Z_{n}^{(G)}$ directly related to $N-2$ poles $P_{m}^{(\Sigma)}$. Clearly, when the spectral weight of the atomic excitation becomes small, the corresponding $P_{n}^{(G)}$ becomes close to $Z_{n}^{(G)}$ and the cancellation occurs. Therefore in realistic situations when only the upper and lower Hubbard bands have significant spectral weight along with the quasiparticle peak, the actual degree of the polynomial expansion is either two or three. However, it is advantageous numerically and cheap computationally to keep all poles and zeroes in Eq. (6) because the formula 
automatically distributes spectral weight over all existing Hubbard bands.

In the limit when $U \rightarrow 0$ the self-energy automatically translates to the non-interacting one. The atomic poles get close to each other but, most importantly, their spectral weight goes rapidly to zero as it gets accumulated within the quasiparticle band.

In the Mott insulating regime, the conditions $b$ ) and c) drop out while all poles $P_{n}^{(G)}$ and zeroes $Z_{n}^{(G)}$ can be used to determine the interpolation. However, in this regime it does not matter whether one of $Z_{n}^{(G)}$ closest to $\omega=0$ is dropped out or kept, since we can always replace this information by information about $\Sigma(\infty)$. Therefore the Mott transition can be studied without changing the constraints.

We thus see that in the insulating case the self-energy correctly reproduces the well-known result of the Hubbard I method where the Green function is computed after Eq. (9) with atomic self-energy. If the lifetime effects are computed, the parameters $P_{n}^{(G)}$ and $Z_{n}^{(G)}$ become complex and the Hubbard bands will acquire an additional bandwidth. This effect is evident from the simulations using various perturbative or QMC impurity solvers and can be naturally incorporated into the interpolative formulas (2) or (6). However, in practical implementation below we will omit it for illustrative purposes.

Let us now discuss the quality of interpolation from the perspective of the high-frequency behavior for the self-energy. The latter can be viewed ${ }^{30}$ as expansion in terms of the moments $\Sigma^{(m)}$, i.e.,

$$
\Sigma(\omega \rightarrow \infty)=\sum_{m=0}^{\infty} \frac{\Sigma^{(m)}}{\omega^{m}},
$$

Most important for us is to look at highest moments which are given by the Hartree Fock value, Eq. (7) involving single occupancy matrix $\langle n\rangle$, as well as the first moment

$\Sigma^{(1)}=\left[(N-1)(N-2)\langle n n\rangle+(N-1)\langle n\rangle-(N-1)^{2}\langle n\rangle^{2}\right] U^{2}$,

containing a double occupancy matrix $\langle n n\rangle$. We see that the interpolation in part relies on the accuracy in computing multiple occupancies which are the functionals of both atomic excitations and the hybridization function. In this regard, using exact atomic Green function to find poles $P_{n}^{(G)}$ and zeroes $Z_{n}^{(G)}$ as part of the constrained procedure may not be as accurate since it would assume the use of atomic multiple occupancies which do not carry information about $\Delta(\omega)$. On the other hand, we can also use only a functional form of the atomic Green function where the multiple occupancies are computed in a more rigorous manner. In the next Section we will show how this can be implemented using the SBMF multiple occupancies which will be found to be in better agreement with the quantum Monte Carlo data.
Note that the moments $\Sigma^{(m)}$ themselves can be used in establishing the constraints for interpolation coefficients. This would involve independent evaluations of $\langle n\rangle,\langle n n\rangle,\langle n n n\rangle$, etc. as well as various integrals involving hybridization function $\Delta(\omega)$. However, we may run into ill-defined numerical problem since high-frequency information will be used to extract the low-frequency behavior. Therefore, it is more advantageous numerically to use some poles and zeros of $G^{a t}(\omega)$ as given by condition d) above.

We thus see that the interpolational scheme is defined completely once a prescription for obtaining parameters such as $\Sigma(0), z,\langle n\rangle$ as well as poles $P_{n}^{(G)}$, and zeroes $Z_{n}^{(G)}$ is given. For this purpose we will test two commonly used methods: SBMF method due to Gutzwiller ${ }^{22}$ as described by Kotliar and Ruckenstein ${ }^{23}$ and the wellknown Hubbard I approximation ${ }^{26}$. We compare these results against more accurate but computationally demanding quantum Monte Carlo calculations and establish the procedure to extract all necessary parameters.

Note that once the constraints such as $z$ are computed from a given approximate method, some of the quantities such as the total number of particles, $\langle n\rangle$, and the value of the self-energy at zero frequency, $\Sigma(0)$, can be computed fully self-consistently. They can be compared with their non-self-consistent values. If the approximate scheme already provides a good approximation for $\langle n\rangle$ and satisfies the Friedel sum rule, the self-consistency can be avoided hence accelerating the calculation. Indeed we found that inclusion of the self-consistency improves the results only marginally except when we are in a close vicinity to the Mott transition but here we do not expect that our simple interpolative algorithm is very accurate.

We now give the description of the approximate methods for solving the impurity model and then present the comparisons of our interpolative procedure with the QMC calculations.

\section{METHODS FOR SOLVING IMPURITY MODEL}

\section{A. Quantum Monte Carlo Method}

The quantum Monte Carlo method is a powerful and manifestly not perturbative approach in either interaction $U$ or the bandwidth $W$. In the QMC method one introduces a Hubbard-Stratonovich field and averages over it using the Monte Carlo sampling. This is a controlled approximation using different expansion parameter, the size of the mesh for the imaginary time discretization. Unfortunately it is computationally very expensive as the number of time slices and the number of HubbardStratonovich fields increases. Also the method works best at imaginary axis while analytical continuation is less accurate and has to be done with a great care. Extensive description of this method can be found in Ref. 1. We 
will use this method to benchmark our calculations using approximate algorithms described later in this Section.

\section{B. Slave-Boson Mean Field Method}

A fast approach to solve a general impurity problem is the slave-boson method 23.24 .25 . At the mean field level, it gives the results similar to the famous Gutzwiller approximation $^{22}$. However, it is improvable by performing fluctuations around the saddle point. This approach is accurate as it has been shown recently to give the exact critical value of $U$ in the large degeneracy limit at half-filling 28 .

The main idea is to rewrite atomic states consisting of $n$ electrons $\left|\gamma_{1}, \ldots, \gamma_{n}\right\rangle, 0 \leq n \leq N$ with help of a set of slave-bosons $\left\{\psi_{n}^{\gamma_{1}, \ldots, \gamma_{n}}\right\}$. In the following, we assume $S U(N)$ symmetric case, i.e., equivalence between different states $\left|\gamma_{1}, \ldots, \gamma_{n}\right\rangle$ for fixed $n$. Formulae corresponding to a more general crystal-field case are given in Appendix B. The creation operator of a physical electron is expressed via slave particles in the standard manner ${ }^{24}$. In order to recover the correct non-interacting limit at the mean-field level, the Bose fields $\psi_{n}$ can be considered as classical values found from minimizing a Lagrangian $L\left\{\psi_{n}\right\}$ corresponding to the Hamiltonian (11). Two Lagrange multipliers $\lambda$ and $\Lambda$ should be introduced in this way, which correspond to the following two constrains:

$$
\begin{aligned}
& \sum_{n=0}^{N} C_{n}^{N} \psi_{n}^{2}=1, \\
& \sum_{n=0}^{N} n C_{n}^{N} \psi_{n}^{2}=T N \sum_{i \omega} G_{g}(i \omega) e^{i \omega 0^{+}}=\bar{n} .
\end{aligned}
$$

The numbers $\psi_{n}^{2}$ are similar to the probabilities $X_{n}$ discussed in connection to the formula for the atomic Green function (3). We thus see the physical meaning of the first constrain which is the sum of probabilities to find atom in any state is equal to one, and the second constrain gives the mean number of electrons coinciding with that found from $G_{g}(\omega)=\left(\omega-\lambda-b^{2} \Delta(i \omega)\right)^{-1}$. A combinatorial factor $C_{n}^{N}=\frac{N !}{n !(N-n) !}$ arrives due to assumed equivalence of all states with $n$ electrons.

Minimization of $L\left\{\psi_{n}\right\}$ with respect to $\psi_{n}$ leads us to the following set of equations to determine the quantities $\psi_{n}$ :

$$
\begin{gathered}
{\left[E_{n}+\Lambda-n \lambda\right] \psi_{n}+n b T \sum_{i \omega} \Delta(i \omega) G_{g}(i \omega)\left[L R \psi_{n-1}+\psi_{n} b L^{2}\right]+} \\
+(N-n) b T \sum_{i \omega} \Delta(i \omega) G_{g}(i \omega)\left[R^{2} b \psi_{n}+L R \psi_{n+1}\right]=0
\end{gathered}
$$

where $b=R L \sum_{n=1}^{N} C_{n-1}^{N-1} \psi_{n} \psi_{n-1}$, determines the mass renormalization, and the coefficients $L=(1-$

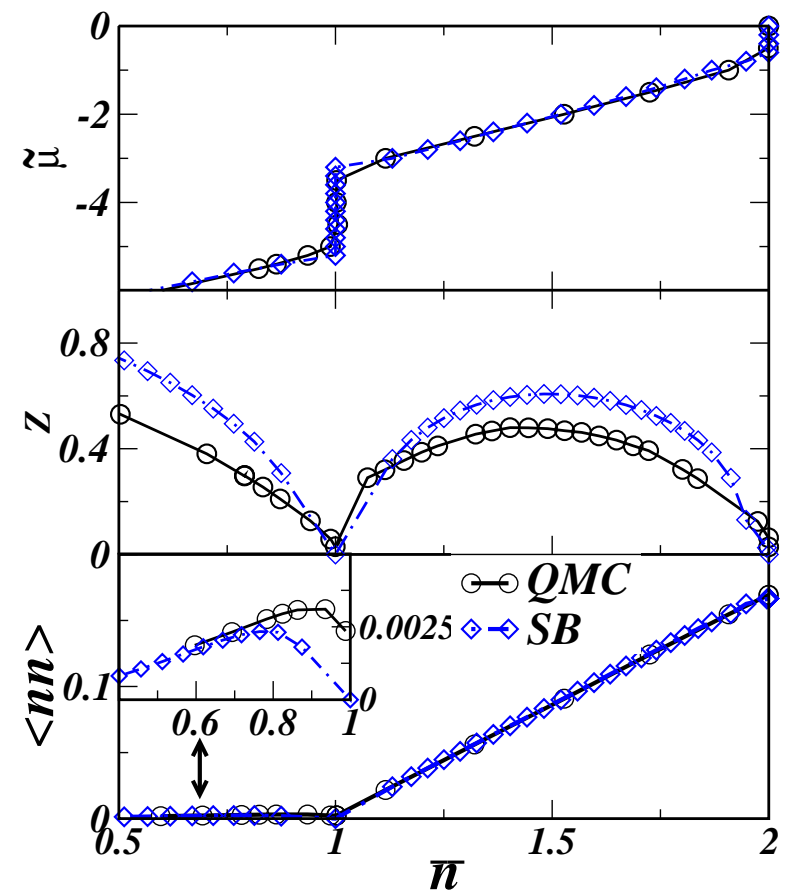

FIG. 1: Comparison between the slave boson mean field and the QMC calculation for (a) concentration versus chemical potential $\tilde{\mu}=\mu-\epsilon_{f}-(N-1) U / 2$, (b) dependence of the spectral weight $Z$ on concentration, and (c) density-density correlation function, $\langle n n\rangle$ versus filling, $\bar{n}$, in the two-band Hubbard model in $S U(4)$ and $U=4=2 W$.

$\left.\sum_{n=1}^{N} C_{n-1}^{N-1} \psi_{n}^{2}\right)^{-1 / 2}, R=\left(1-\sum_{n=0}^{N} C_{n}^{N-1} \psi_{n}^{2}\right)^{-1 / 2}$ are normalization constants as in Refs. 23 24. $E_{n}=\epsilon_{f} n+$ $U n(n-1) / 2$ is the total energy of the atom with $n$ electrons in $S U(N)$ approximation.

Eq. (23), along with the constrains (21), (22) constitute a set of non-linear equations which have to be solved iteratively. In practice, we consider Eq. (23) as an eigenvalue problem with $\Lambda$ being the eigenvalue and $\psi_{n}$ being the eigenvectors of the matrix. The physical root corresponds to the lowest eigenvalue of $\Lambda$ which gives a set of $\psi_{n}$ determining the mass renormalization $Z=b^{2}$. Since the matrix to be diagonalized depends non-linearly on $\psi_{n}$ via the parameters $L, R$, and $b$ and also on $\lambda$, the solution of the whole problem assumes the self-consistency: i) we build an initial approximation to $\psi_{n}$ (for example the Hartree-Fock solution) and fix some $\lambda$, ii) we solve eigenvalue problem and find new normalized $\psi_{n}$, iii) we mix new $\psi_{n}$ with the old ones using the Broyden $\operatorname{method}^{29}$ and build new $L, R$, and $b$. Steps ii) and iii) are repeated until the self-consistency with respect to $\psi_{n}$ is reached. During the iterations we also vary $\lambda$ to obey the constrains. The described procedure provides a stable computational algorithm for solving AIM and gives us an access to the low-frequency Green's function and the self-energy of the problem via knowledge of the slope of $\Im \Sigma(i \omega)$ and the value $\Re \Sigma(0)$ at zero frequency.

The described slave-boson method gives the following 
expression for the self-energy:

$$
\Sigma(\omega)=\left(1-b^{-2}\right) \omega-\epsilon_{f}+\lambda b^{-2} .
$$

The impurity Green function $G_{f}(\omega)$ in this limit is given by the expression

$$
G_{f}(\omega)=b^{2} G_{g}(\omega)
$$

As an illustration, we now give the solution of Eq. (23) for non-degenerate case $(N=2)$ and at the particlehole symmetry point with $\epsilon_{f}-\mu=-\frac{U}{2}(N-1)$. Consider a dynamical mean-field theory for the Hubbard model which reduces the problem to solving the impurity model subject to the self-consistency condition with respect to $\Delta(\omega)$. Starting with the semicircular density of states (DOS), the self-consistency condition is given by Eq. (23). We obtain the following simplifications: $L=R=\sqrt{2}, \lambda=0, \psi_{0}=\psi_{2}, b=4 \psi_{1} \psi_{2}$ and $G_{g}(\omega)=$ $\left[\omega-\left(\frac{W}{4}\right)^{2} b^{2} G_{g}(\omega)\right]^{-1}$. The sum $T \sum_{i \omega} \Delta(i \omega) G_{g}(i \omega)$ appeared in Eq. (23) scales as $W \alpha / 2$ with the constant $\alpha$ being the characteristic of a particular density of states and approximately equal to -0.2 in the semicircular DOS case. The self-consistent solution of Eq. (23) is therefore possible and simply gives $\psi_{2}^{2}=\frac{U}{32 W \alpha}+\frac{1}{4}$. The Mott transition occurs when no sites with double occupancies can be found, i.e. when $\psi_{0}=\psi_{2}=0$. A critical value of $U_{c}=8 W|\alpha|$. For $\alpha \approx-0.2$, this gives $U_{c} \approx 1.6 \mathrm{~W}$ and reproduces the result for $U_{c 2}=1.49 \mathrm{~W}$ known from the QMC calculation within a few percent accuracy. As degeneracy increases, critical $U$ is shifted towards higher values ${ }^{28}$. From numerical calculations we obtained the following values of the critical interactions in the halffilled case $U_{c} \approx 3 W$ for $N=6$ ( $p$-level), $U_{c} \approx 4.5 W$ for $N=10(d$-level $)$, and $U_{c} \approx 6 W$ for $N=14(f$-level $)$.

Density-density correlation function $\langle n n\rangle$ for local states with $n$ electrons is proportional to the number of pairs formed by $n$ particles $C_{2}^{n} / C_{2}^{N}$. Since the probability for $n$ electrons to be occupied is given by: $P_{n}=\psi_{n}^{2} C_{n}^{N}$, the physical density-density correlator can be deduced from: $\langle n n\rangle=\sum_{n} C_{2}^{n} / C_{2}^{N} P_{n}$. Similarly, the triple occupancy can be calculated from $\langle n n n\rangle=\sum_{n} C_{3}^{n} / C_{3}^{N} P_{n}$.

Let us now check the accuracy of this method by comparing its results with the QMC data. We consider the two-band Hubbard model in $S U(N=4)$ orbitally degenerate case. Hybridization $\Delta(\omega)=\sum_{\mathbf{k}} V(\mathbf{k}) /\left(\omega-E_{\mathbf{k}}\right)$ satisfies the DMFT self-consistency condition of the Hubbard model on a Bethe lattice

$$
\Delta(\omega)=\left(\frac{W}{4}\right)^{2} G(\omega)
$$

The Coulomb interaction is chosen to be $U=2 W$ which is sufficiently large to open the Mott gap at integer fillings. All calculations are done for the temperature $T=1 / 32 W$.

We first compare the average number of electrons vs. chemical potential determined from the slave bosons which is plotted in Fig. 1(a). This quantity is sensitive to the low-frequency part of the Green function which should be described well by the present method. We see that it reproduces the QMC data with a very high accuracy and only differs by 20 per cent very near the jump of $\tilde{\mu}$ at $n=1$.

The quasiparticle residue $z$ versus filling $\bar{n}$ is plotted in Fig. 1(b). The slave-boson method gives the Fermi liquid and provides estimates for the quasiparticle residue with the overall discrepancy of the order of $30 \%$. In fact, we have performed several additional calculations for other degeneracies $(N=2$ and 6$)$ and for various parameters regimes. The trend to overestimate mass renormalization can be seen in many cases. It disappears only when $U$ approaches zero. We need to point out, however, that i) the extractions of zero frequency self-energy slopes from the high-temperature QMC is by itself numerically not well grounded procedure, as information for the self-energy is known at the Matsubara points only, which is then extrapolated to $\omega=0$, ii) other methods for solving impurity model, such as NCA or IPT display similar discrepancies and iii) recent findings 28 suggest that at least at half-filling quasiparticle residues deduced from slave bosons becomes exact when $N \rightarrow \infty$. Most importantly for our interpolative method is that the entire functional dependence of $z$ vs. filling, interaction and degeneracy is correctly captured. Its overall accuracy is acceptable as it is evident from our comparisons of the spectral functions presented in the next Section and well within the main goal of our work to provide fast scans of the entire parameter space necessary for simulating real materials. This is important as, for example, for general $f$-electron material, the QMC method is prohibitively time consuming, but we expect from the SBMF method the results for mass renormalization not worse than $50 \%$ for such delicate regime as the vicinity of the Mott transition.

Fig. 1(c) shows the density-density correlation function $\langle n n\rangle$ as a function of average occupation $\bar{n}$. The discrepancy is most pronounced for fillings $\bar{n}<1$ [see the inset of Fig. 1(c)] where the absolute values of $\langle n n\rangle$ are rather small. Although our slave-boson technique captures only the quasiparticle peak, it gives the correlation function in reasonable agreement with the QMC for dopings not too close to the Mott transition.

\section{Hubbard I Approximation}

Now we turn to the Hubbard I approximation ${ }^{26}$ which is closely related to the moments expansion method ${ }^{30}$. Consider many-body atomic states $\left|\Phi_{\kappa}^{(n)}\right\rangle$ which in $S U(N)$ are all degenerate with index $\kappa$ numerating these states for a given number of electrons $n$. The impurity Green function is defined as the average

$$
G_{f}(\tau)=-\left\langle T_{\tau} f_{\alpha}(\tau) f_{\beta}^{+}(0)\right\rangle .
$$

and becomes diagonal with all equal elements in $S U(N)$.It is convenient to introduce the Hubbard opera- 
tors

$$
\hat{X}_{\kappa \kappa^{\prime}}^{n n^{\prime}}=\left|\Phi_{\kappa}^{(n)}\right\rangle\left\langle\Phi_{\kappa^{\prime}}^{\left(n^{\prime}\right)}\right|
$$

and represent the one-electron creation and destruction operators as follows

$$
\begin{aligned}
f_{\alpha} & =\sum_{n} \sum_{\kappa \kappa^{\prime}}\left\langle\Phi_{\kappa}^{(n)}\left|f_{\alpha}\right| \Phi_{\kappa^{\prime}}^{(n+1)}\right\rangle \hat{X}_{\kappa \kappa^{\prime}}^{n n+1}, \\
f_{\alpha}^{+} & =\sum_{n} \sum_{\kappa \kappa^{\prime}}\left\langle\Phi_{\kappa}^{(n+1)}\left|f_{\alpha}^{+}\right| \Phi_{\kappa^{\prime}}^{(n)}\right\rangle \hat{X}_{\kappa \kappa^{\prime}}^{n+1 n} .
\end{aligned}
$$

The impurity Green function (27) is given by

$$
G_{f}(\tau)=\sum_{n m} G_{n m}(\tau)
$$

where the matrix $G_{n m}(\tau)$ is defined as

$$
G_{n m}(\tau)=-\sum_{\kappa_{1} \kappa_{2} \kappa_{3} \kappa_{4}}\left\langle\Phi_{\kappa_{1}}^{(n)}\left|f_{\alpha}\right| \Phi_{\kappa_{2}}^{(n+1)}\right\rangle\left\langle T_{t} \hat{X}_{\kappa_{1} \kappa_{2}}^{n n+1}(\tau) \hat{X}_{\kappa_{3} \kappa_{4}}^{m+1 m}(0)\right\rangle\left\langle\Phi_{\kappa_{3}}^{(m+1)}\left|f_{\alpha}^{+}\right| \Phi_{\kappa_{4}}^{(m)}\right\rangle .
$$

Establishing the equations for $G_{n m}(\tau)$ can be performed using the method of equations of motion for the $X$ operators. Performing their decoupling due to Hubbard26.31, carrying out the Fourier transformation and analytical continuation to the real frequency axis, and summing over $n$ and $m$ after (31) we arrive to the net result

$$
G_{f}^{-1}(\omega)=G_{a t}^{-1}(\omega)-\Delta(\omega)
$$

The $G_{a t}(\omega)$ can be viewed in the matrix form (31) with the following definition of a diagonal atomic Green function

$$
G_{n m}^{a t}(\omega)=\delta_{n m} \frac{C_{n}^{N-1}\left(X_{n}+X_{n+1}\right)}{\omega+\mu-E_{n+1}+E_{n}} .
$$

with $E_{n}=\epsilon_{f} n+U n(n-1) / 2$ being the total energies of the atom with $n$ electrons in $S U(N)$. The coefficients $X_{n}$ are the probabilities to find atom with $n$ electrons and were already discussed in connection to the formula (3) for the atomic Green function. They are similar to the coefficients $\psi_{n}^{2}$ introduced within the SBMF method but now found from different set of equations. These numbers are normalized to unity, $\sum_{n=0}^{N} C_{n}^{N} X_{n}=$ $\sum_{n=0}^{N-1} C_{n}^{N-1}\left(X_{n}+X_{n+1}\right)=1$, and are expressed via diagonal elements of $G_{n m}(i \omega)$ as follows:

$$
X_{n}=-T \sum_{i \omega} G_{n n}(i \omega) e^{-i \omega 0^{+}} / C_{n}^{N-1}
$$

Their determination in principle assumes solving a non-linear set of equations while determining $G_{f}(\omega)$. The mean number of electrons can be measured as follows: $\quad \bar{n}=\sum_{n=0}^{N} n C_{n}^{N} X_{n}$ or as follows $\bar{n}=$ $T N \sum_{i \omega} G_{f}(i \omega) e^{i \omega 0^{+}}$. The numbers $X_{n}$ can be also used to find the averages $\langle n n\rangle,\langle n n n\rangle$ in the way similar to what has been done in the SBMF approach.

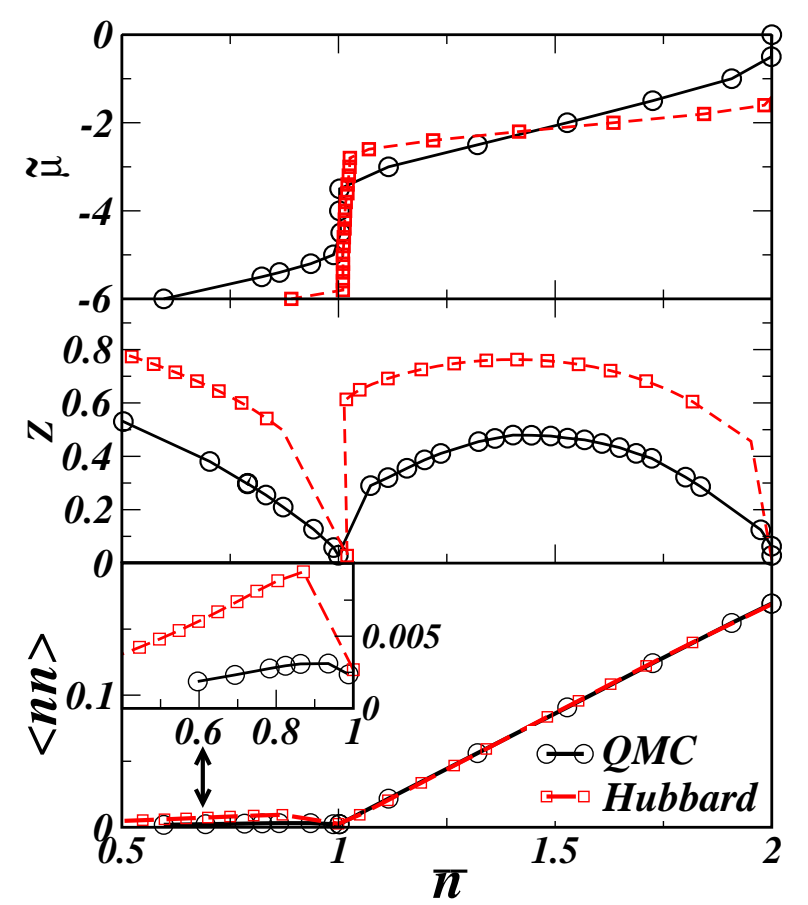

FIG. 2: Comparison between the Hubbard I and the QMC calculation for (a) concentration versus chemical potential $\tilde{\mu}=\mu-\epsilon_{f}-(N-1) U / 2$, (b) dependence of the spectral weight $Z$ on concentration, and (c) density-density correlation function, $\left\langle n_{\alpha} n_{\alpha^{\prime}}\right\rangle$ versus filling, $\bar{n}$, in the two-band Hubbard model in $S U(4)$ and for $U=2 W=4$.

If we neglect by the hybridization $\Delta(\omega)$ in Eq. (33), the probabilities $X_{n}$ become simply statistical weights:

$$
X_{n}=\frac{e^{-E_{n} / T}}{\sum_{m=0}^{N} C_{m}^{N} e^{-E_{m} / T}} .
$$

We thus see that in principle there are several different ways to determine the coefficients $X_{n}$, either via selfconsistent determination (35), or using statistical formula (36), or taking them from SBMF equation (23), 
i.e. setting $X_{n}=\psi_{n}^{2}$ while still utilizing the functional dependence provided by the Hubbard I method. To determine the best procedure let us first consider limits of large and small $U$ 's. When $\Delta(\omega) \equiv 0, G_{f}(\omega)$ is reduced to $\sum_{n m} G_{n m}^{a t}(\omega)$, i.e. the Hubbard I method reproduces the atomic limit. Setting $U \equiv 0$ gives $G_{f}(\omega)=\left[\omega+\mu-\epsilon_{f}-\Delta(\omega)\right]^{-1}$, which is the correct band limit. Unfortunately, at half-filling this limit has a pathology connected to the instability towards Mott transition at any interaction strength $U$. To see this, we consider a dynamical mean-field theory for the Hubbard model. Using semicircular density of states, we obtain $G_{f}(\omega)=\left[1-\left(\frac{W}{4}\right)^{2} G_{f}(\omega) G_{a t}(\omega)\right]^{-1} G_{a t}(\omega)$ and conclude that for any small $U$ the system opens a pathological gap in the spectrum. Clearly, using Hubbard I only, the behavior of the Green function at $\omega \rightarrow 0$ cannot be reproduced and the quality of the numbers $X_{n}$ is at question. This already emphasizes the importance of using the slave-boson treatment at small frequencies.

Ultimately, making the comparisons with the QMC calculations is the best option in picking the most accurate procedure to compute the probabilities $X_{n}$. To check the accuracy against the QMC we again consider the twoband Hubbard model in $S U$ (4) symmetry as above. The chemical potential, mass renormalization and double occupancy are plotted versus filling in Fig. 2 All quantities here were computed with statistical weights after Eq. (36) but we found a similar agreement while utilizing the self-consistent determination of $X_{n}$ after Eq. (35). We first see that the Hubbard I approximation does not give satisfactory agreement with the QMC data for $\bar{n}(\tilde{\mu})$ because it misses the correct behavior at low frequencies.

The comparisons for $z(\bar{n})$ plotted in Fig. 2(b) surprisingly show a relatively good behavior. However, the pathology of this approximation at half-filling would predict $z=0$ for any $U$, which is a serious warning not to use it for extracting the quasiparticle weight. Fig. 22(c) shows $\langle n n\rangle$ as a function of average occupation $\bar{n}$. As this quantity is directly related to the high-frequency expansion one may expect a better accuracy here. However, comparing Fig. 2(c) and Fig. 1(c), it is clear that the slave boson method gives more accurate double occupancy. This is due to the fact that the density matrix obtained by the slave boson method is of higher quality than the one obtained from the Hubbard I approximation.

The results of these comparisons suggest that the probabilities $\psi_{n}^{2}$ provided by the slave-boson method is a better way in determining the coefficients $X_{n}$ in the metallic region of parameters. Therefore it is preferable to use these numbers while establishing the equations for the unknown coefficients in the interpolational form (2). However, the functional form (34) of the Hubbard I approximation with $X_{n}=\psi_{n}^{2}$ can still be used as it provides the positions of the poles $P_{n}^{(G)}$ and zeroes $Z_{n}^{(G)}$ of the atomic Green function necessary for the condition d) in the previous Section. This also ensures accurate high-frequency behavior of the interpolated self-energy since its moments expressed via multiple occupancies are directly related to $\psi_{n}^{2}$.

Interestingly, while more sophisticated QMC approach captures both the quasiparticle peak and the Hubbard bands this is not the case for the slave-boson mean field method. To obtain the Hubbard bands in this method fluctuations need to be computed, which would be very tedious in a general multiorbital situation. However the slave-boson method delivers many parameters in a good agreement with the QMC results, and, hence, it can be used to give a functional dependence of the coefficients of the rational approximation.

\section{RESULTS OF THE INTERPOLATIVE SCHEME}

By now the procedure to determine the coefficients is well established. We use the SBMF method to determine $\bar{n}, \Sigma(\infty), \Sigma(0), z$ as well as poles and zeroes of the atomic Green function provided by the SBMF probabilities $\psi_{n}^{2}$ and by the bare atomic energy levels $E_{n}$ (we omit the lifetime effects for simplicity). This generates a set of linear equations for coefficients $a_{m}^{\alpha}, b_{m}^{\alpha}$ in the rational interpolation formula (2). In the present Section we show the trends our interpolative algorithm gives for the spectral functions in various regions of parameters as well as provide detailed comparisons for some values of doping for both imaginary and real axis spectral functions. The two-band Hubbard model with semicircular density of states and DMFT self-consistency condition after (26) is utilized in $S U(N=4)$ symmetry in all cases using the bandwidth $W=2$ and temperature $T=1 / 16$.

\section{A. Trends}

Fig. 3] shows the behavior of the density of states $N(\omega)=-\Im G_{f}(\omega) / \pi$ for $U=W$ as a function of the chemical potential $\tilde{\mu}$ computed with respect to the particle-hole symmetry point $(N-1) U / 2$ and as a function of frequency $\omega$. The semicircular quasiparticle band is seen at the central part of the figure. Its bandwidth is only weakly renormalized by the interactions in this regime. It is half-filled for $\tilde{\mu}=0$ (i.e. when $\left.\mu=\epsilon_{f}-(N-1) U / 2\right)$ and gets fully emptied when chemical potential is shifted to negative values. Several weak satellites can be also seen on this figure which are due to atomic poles. Their spectral weight is extremely small in this case and any sizable lifetime effect (which is not included while plotting this figure) will smear these satellites out almost completely. While approaching fully emptied (or fully filled situation) the spectral weight of the Hubbard bands disappears completely and only unrenormalized quasiparticle band remains. It is clear that even without shifting the atomic poles to the complex axis, the numerical procedure of generating the selfenergy is absolutely stable. 


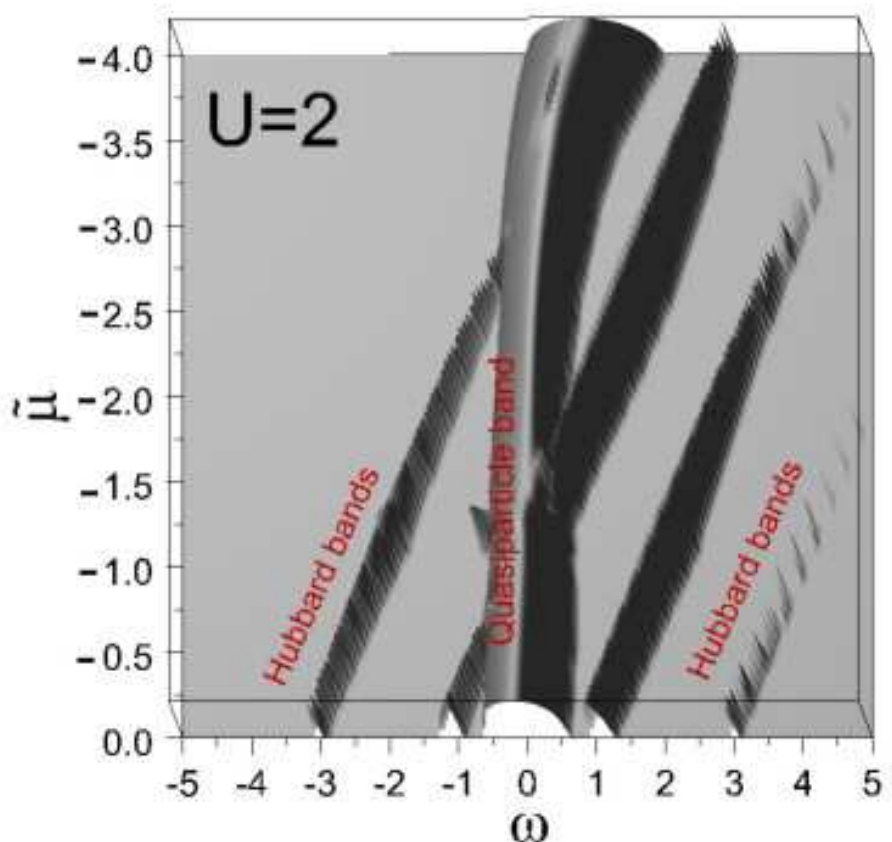

FIG. 3: Calculated density of states using the interpolative method as a function of chemical potential $\tilde{\mu}=\mu-\epsilon_{f}-$ $(N-1) U / 2$ and frequency for the two-band Hubbard model in $S U(4)$ and at $U=W=2$.

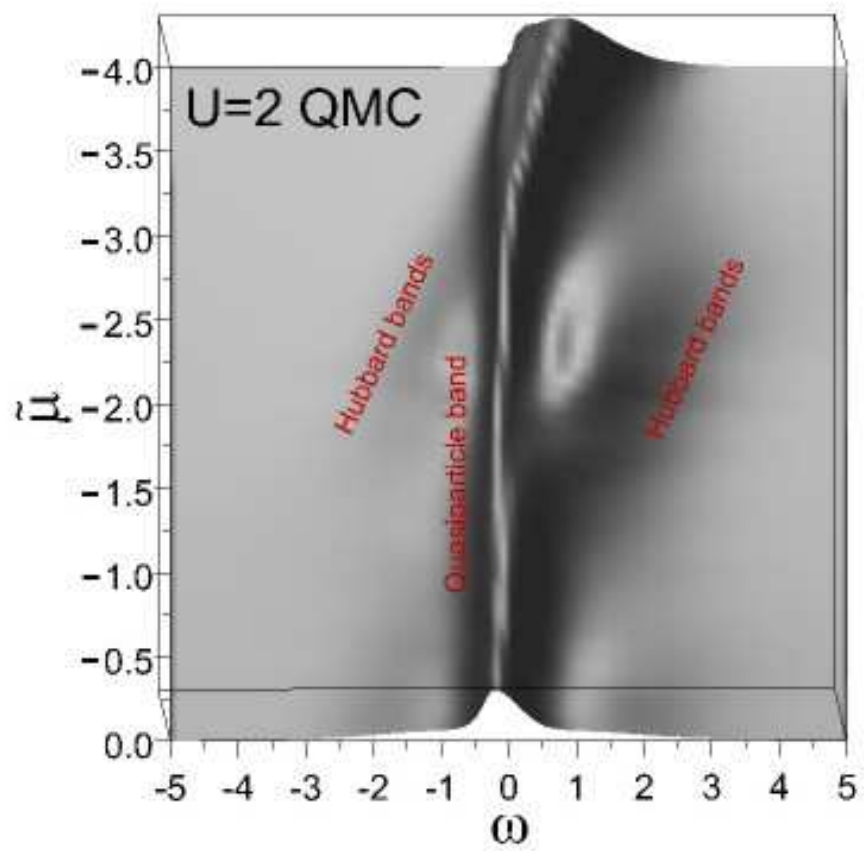

FIG. 4: Calculated density of states using the QMC method as a function of chemical potential $\tilde{\mu}=\mu-\epsilon_{f}-(N-1) U / 2$ and frequency for the two-band Hubbard model in $S U(4)$ and at $U=W=2$.

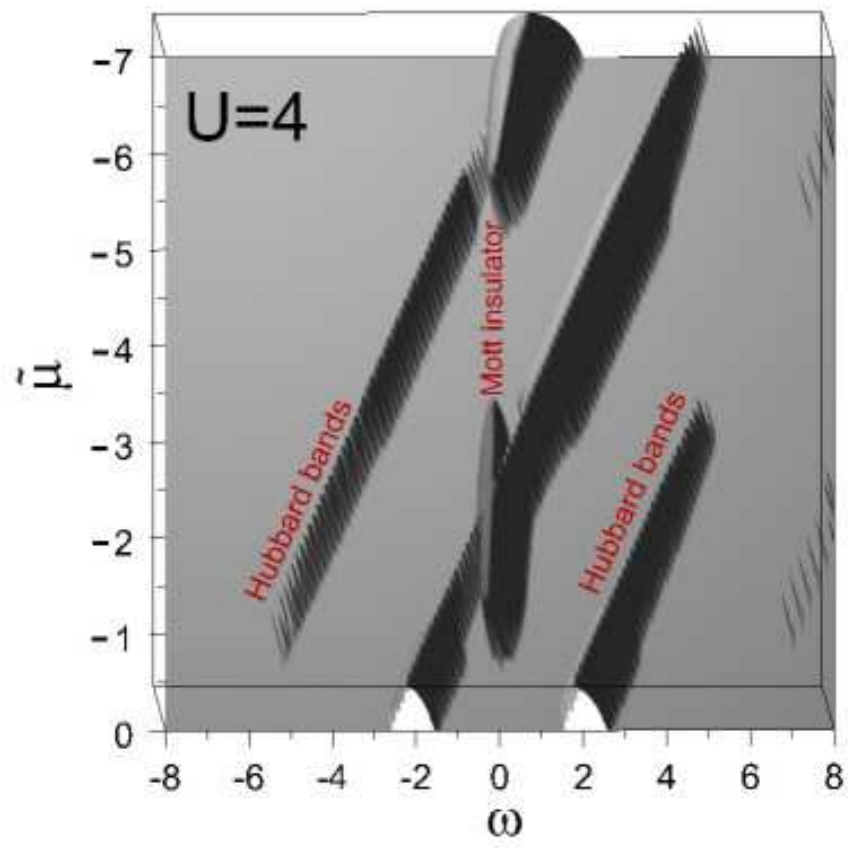

FIG. 5: Calculated density of states using the interpolative method as a function of chemical potential $\tilde{\mu}=\mu-\epsilon_{f}-$ $(N-1) U / 2$ and frequency for the two-band Hubbard model in $S U(4)$ and at $U=2 W=4$.

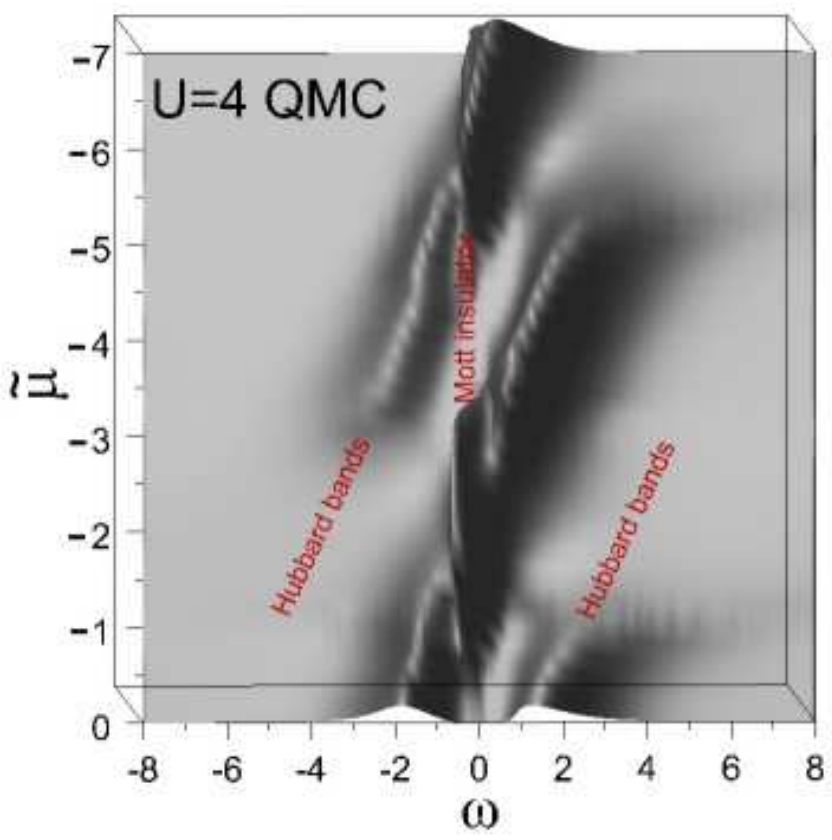

FIG. 6: Calculated density of states using the QMC method as a function of chemical potential $\tilde{\mu}=\mu-\epsilon_{f}-(N-1) U / 2$ and frequency for the two-band Hubbard model in $S U(4)$ and at $U=2 W=4$. 
This trend can be directly compared with the simulations using a more accurate QMC impurity solver. We present this in Fig. 4 for $U=W$, which shows calculated density of states in the same region of parameters. Remarkably that again we can distinguish the renormalized quasiparticle band and very weak Hubbard satellites. The Hubbard bands appear to be much more diffuse in this figure mainly due to the lifetime effects and partially due to maximum entropy method using for analytical continuation from imaginary to real axis. Otherwise the entire picture looks very much like the one on Fig. 3. generated with much less computational effort.

Fig. [5] gives the same behavior of the density of states for the strongly correlated regime $U=2 W$. In this case the situation at integer filling is totally different as the system undergoes metal-insulator transition. This is seen around the dopings levels with $\tilde{\mu}$ between 0 and -1 and between -3 and -5 where the wight of the quasiparticle band collapses while lower and upper Hubbard bands acquire all spectral weight. In the remaining region of parameters both strongly renormalized quasiparticle band and Hubbard satellites remain. Again, once full filling or full emptying is approached the quasiparticle bands restores its original bandwidth while the Hubbard bands disappear. The QMC result for the same region of parameters is given in Fig. 6] Again we can distinguish the renormalized quasiparticle band and Hubbard satellites as well as the areas of Mott insulator and of strongly correlated metal. The Hubbard bands appear to be more sharp in this figure which signals on approaching the atomic limit.

\section{B. Comparison for Spectral Functions at Imaginary Axis}

We now turn to the comparison of the Green functions and the self-energies obtained using the formulae (9), and (2) respectively against the predictions of the quantum Monte Carlo method. We will report our comparisons for the two-band Hubbard model and sets of dopings corresponding to $\bar{n}=0.5,0.8,1.2,1.5,1.8$ using the value of $U=2 W=4$. Other tests for different degeneracies, doping levels and the interactions have been performed which display similar accuracy.

Fig. 7 shows the comparison between the real and imaginary parts of the Green function obtained by the interpolative method with the results of the QMC calculations. As one can see almost complete agreement has been obtained for a wide regime of dopings. The agreement gets less accurate once the half-filling is approached, but still very good giving an extraordinary computational speed of the given method compared to QMC.

Fig. 8] shows similar comparison between the real and imaginary parts of the self-energies obtained by the interpolative and the QMC method. We can see that the selfenergies exhibit some noise which is intrinsic to stochas-

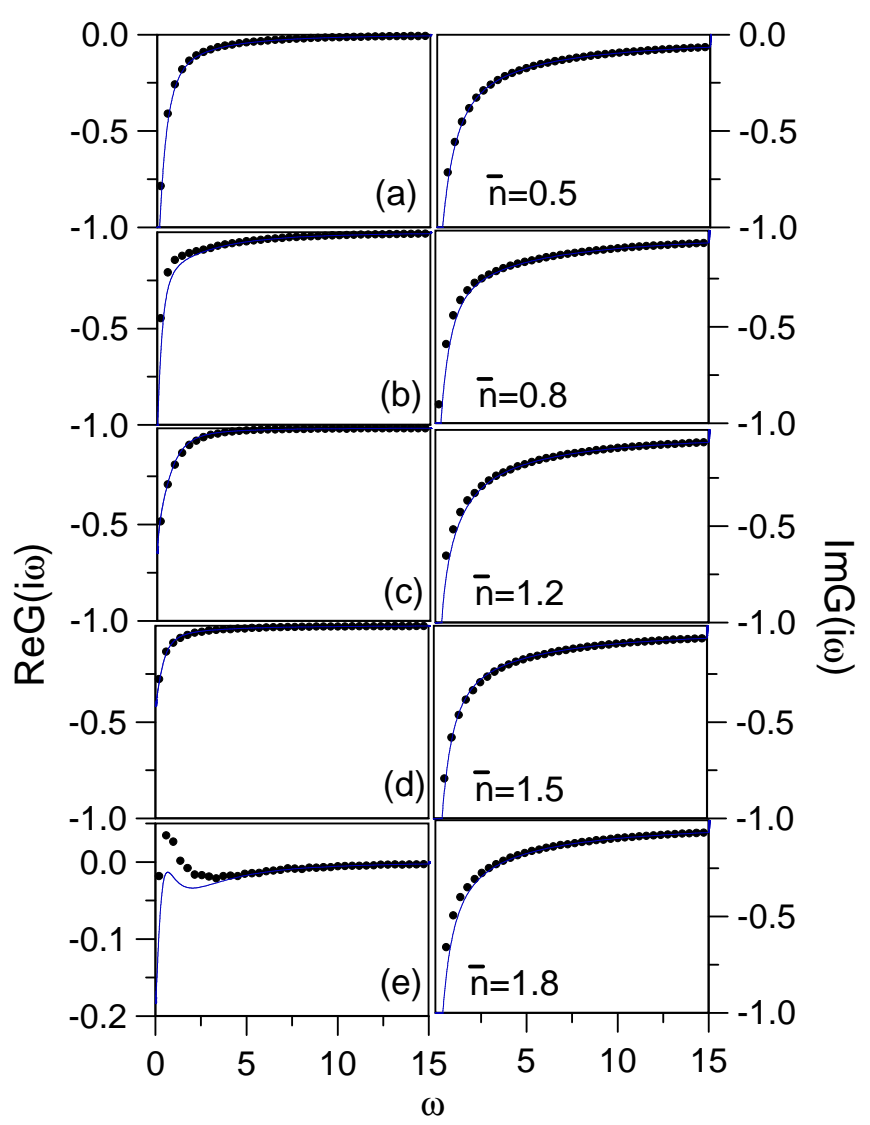

FIG. 7: Comparison between real and imaginary parts of the Green function obtained from the interpoaltive method and the quantum Monte Carlo calculation for the two-band Hubbard model at a set of fillings $\bar{n}=0.5,0.8,1.2,1.5,1.8$ and $U=2 W=4$.

tic QMC procedure. The values of the self-energies near $i \omega=0$ and $i \omega=\infty$ are correctly captured with some residual discrepancies are attributed to slightly different chemical potentials used to reproduce given filling within every method. The results at the imaginary axis show slightly underestimated slopes of the self-energies within the interpolative algorithm which is attributed to the underestimated values of $z$ obtained from the SBMF calculation. Ultimately improving these numbers by inclusions of fluctuations beyond mean field will further improve the comparisons. However, even at the present stage of the accuracy all functional dependence brought by the SBMF method quantitatively captures the behavior of the self-energy seen from time consuming QMC simulation.

\section{Comparison for Spectral Functions at Real Axis}

We also made detailed comparisons between calculated densities of states obtained at the real axis using the interpolative method and the QMC algorithm. The QMC 


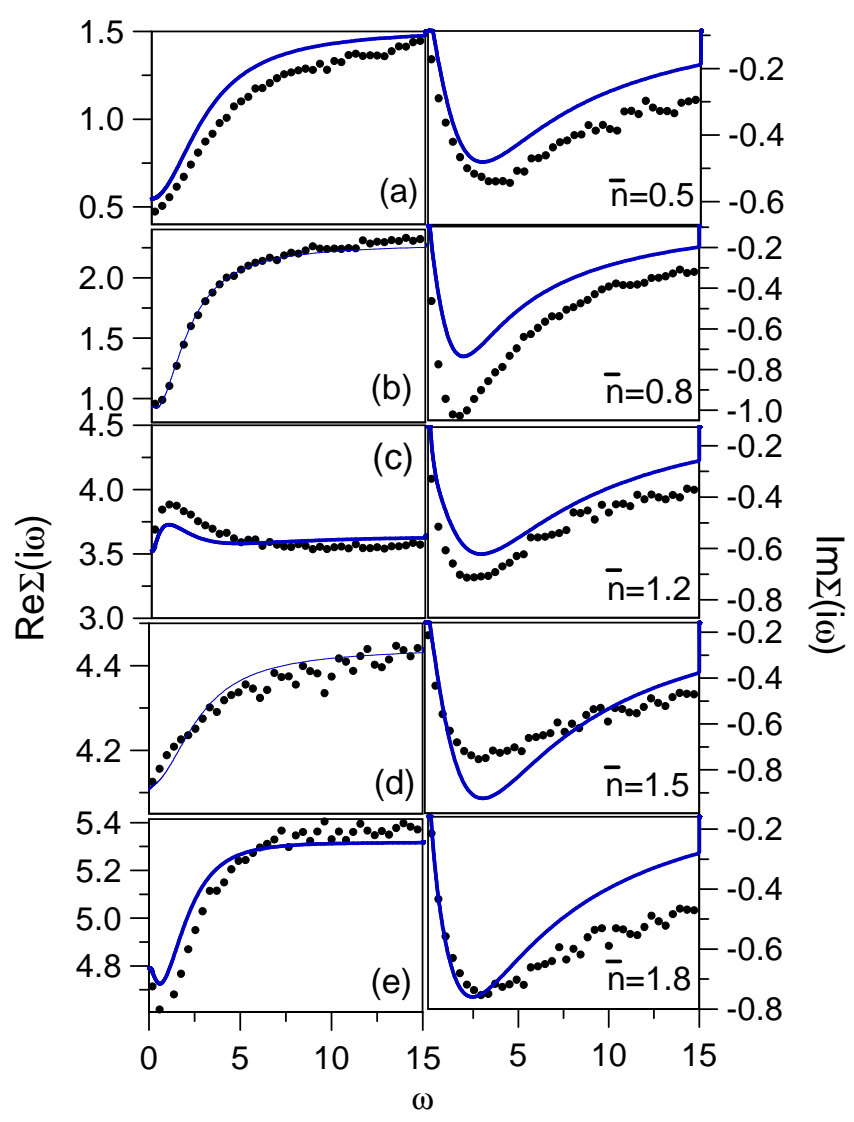

FIG. 8: Comparison between real and imaginary parts of the self-energies obtained from the interpoaltive method and the quantum Monte Carlo calculation for the two-band Hubbard model at a set of fillings $\bar{n}=0.5,0.8,1.2,1.5,1.8$ and $U=$ $2 W=4$.

densities of states require an analytical continuation from the imaginary to real axis and were generated using the maximum entropy method. By itself this procedure introduces some errors within the QMC especially at higher frequencies. In Fig. 9] we show our results for the fillings corresponding to $\bar{n}=0.5,0.8,1.2,1.5,1.8$ using the value of $U=2 W=4$. One can see the appearance of the quasiparticle band and two Hubbard bands distanced by the value of $U$. It can be seen that the interpolative method remarkably reproduces the trend in shifting the Hubbard bands upon changing the doping. It automatically holds the distance between them to the value of $U$ while this is not always true in the quantum Monte Carlo method. Despite this result, the overall agreement between both methods is very satisfactory.

\section{DISCUSSION}

Here we would like to discuss possible ways to further improve the accuracy of the method. The inaccuracies are mainly seen in three different quantities: i) the width of the Hubbard bands, ii) the mass renormalization $z(\mu)$

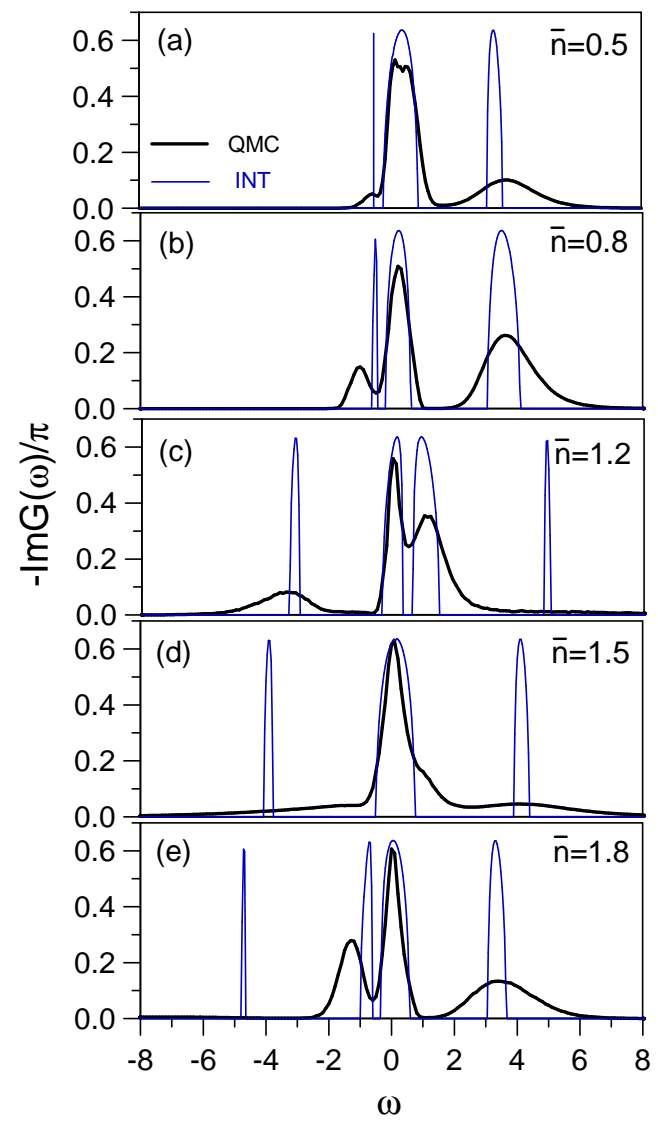

FIG. 9: Comparison between the one-electron densities of states obtained from interpolative formula and the quantum Monte Carlo calculation for the two-band Hubbard model at fillings $\bar{n}=0.5,0.8,1.2,1.5,1.8$. and $U=2 W=4$.

which is borrowed from the SBMF method, and iii) the number of electrons $n(\mu)$ extracted from the interpolated impurity Green function (9). The inaccuracy in the width of the Hubbard band is mainly connected to neglecting the lifetime effect. Provided it is computed, this will shift the positions of atomic poles onto the complex plane which is in principle trivial to account for within our interpolative algorithm. To improve the accuracy of $z(\mu)$ one can, for example, work out a modified slave boson scheme which will account for the fluctuations around mean field solution. The inaccuracy in $n(\mu)$ is small in many regions of parameters and typically amounts to $5-$ 10 per cent. We can try to improve this agreement by requirement that $n(\mu)$ obtained via interpolation matches with $n_{S B M F}(\mu)$ obtained by the SBMF method. The latter agrees very well with the QMC for a wide region of parameters as it is evident from Fig. 1(a). In reality, our analysis shows that in many cases the discrepancy in $n(\mu)$ is connected with the overestimation of $z_{S B M F}(\mu)$. Therefore, points ii) and iii) mentioned above are interrelated.

The requirement that $n(\mu)=n_{S B M F}(\mu)$ can be enforced by adjusting the width of the quasiparticle band, 
and in many regions of parameters this is controlled by $z(\mu)$. However, there are situations when the Hubbard band appears in the vicinity of $\omega=0$, and changing $z(\mu)$ does not affect the bandwidth. To gain a control in those cases it is better to replace the constraints $\Sigma(0), d \Sigma /\left.d \omega\right|_{\omega=0}$ by constraints of fixing the self-energies at two frequencies, say $\Sigma(0)$ and $\Sigma\left(i \omega_{0}\right)$ where $\omega_{0}$ is the frequency of the order of renormalized bandwidth. We have found that this scheme brings mass renormalizations which are about $30 \%$ smaller than the SBMF ones, and the agreement with the QMC is significantly improved. Thus, inaccuracies ii) and iii) can be avoided with this very cheap trick. However, we also would like to point out that the condition $n(\mu)=n_{S B M F}(\mu)$ is essentially non-linear as the solution may not exist for all regions of parameters. It is, for example, evident that in such points where $n(\mu)$ is given by a symmetry (as, e.g., particle-hole symmetry point $n=2$ in the case considered above) the mass renormalization does not affect the number of electrons.

As the philosophy of our approach is to get the best possible fit we are also open to implementing any kinds of ad hoc renormalizations constants. One of such possibility could be the use of a quasiparticle residue 30 per cent smaller than $z_{S B M F}(\mu)$. As $z(\mu)$ should go to unity when $U=0$, the correction can, for example, be encoded into the formula $z(\mu)=z_{S B M F}(\mu)\left[0.7+0.3 z_{S B M F}(\mu)\right]$.

We finally would like to remark that the scheme defined by a set of linear equations for the coefficients (14)-19) is absolutely robust as solutions exist for all regimes of parameters such as strength of the interaction, doping and degeneracy. In general, bringing any information on the self energy $\Sigma\left(\omega_{x}\right)$ at some frequency point $\omega_{x}$ or its derivative $d \Sigma /\left.d \omega\right|_{\omega=\omega_{x}}$ would generate a linear relationship between the interpolation coefficients, thus keeping robustness of the method. On the other hand, fixing such relationships as numbers of electrons brings nonlinearity to the problem which could lead to multiplicity or non-existence of the solutions. It is also clear that by narrowing the regime of parameters, the accuracy of the interpolative algorithm can be systematically increased.

\section{CONCLUSION}

To summarize, this paper shows the possibility to interpolate the self-energies for a whole range of dopings, degeneracies and the interactions using a computationally efficient algorithm. The parameters of the interpolation are obtained from a set of constraints in the slave boson mean field method combined with the functional form of the atomic Green function. The interpolative method reproduces all trends in remarkable agreement with such sophisticated and numerically accurate impurity solver as the QMC method. We also obtain a very good quantitative agreement in a whole range of parameters for such quantities as mean level occupancies, spectral functions and self-energies. Some residual discrepancies remain which can be corrected provided better algorithms delivering the constraints will be utilized. Nevertheless, given the superior speed of the present approach, we have obtained a truly exceptional accuracy times efficiency of the proposed procedure.

The work was supported by NSF-DMR Grants 0096462, 02382188, 0312478, 0342290 and US DOE Grant No DE-FG02-99ER45761. The authors also acknowledge the financial support from the Computational Material Science Network operated by US DOE and from the Ministry of Education, Science and Sport of Slovenia.

\section{APPENDIX}

In the crystal field case we assume that $N$-fold degenerate impurity level $\epsilon_{f}$ is split by a crystal field onto $G$ sublevels $\epsilon_{f 1}, \ldots \epsilon_{f \alpha}, \ldots \epsilon_{f G}$. We assume that for each sublevel there is still some partial degeneracy $d_{\alpha}$ so that $\sum_{\alpha=1}^{G} d_{\alpha}=N$. In limiting case of $S U(N)$ degeneracy, $G=1, d_{1}=N$, and in non-degenerate case, $G=N, d_{1}=d_{\alpha}=d_{G}=1$. We need to discuss how a number of electrons $n$ can be accommodated over different sublevels $\epsilon_{f \alpha}$. Introducing numbers of electrons on each sublevel, $n_{\alpha}$, we obtain $\sum_{\alpha=1}^{G} n_{\alpha}=n$. Note the restrictions: $0<n<N$, and $0<n_{\alpha} \leq d_{\alpha}$. In $S U(N)$ case, $G=1, n_{1}=n$, and in non-degenerate case, $G=N, n_{\alpha}$ is either 0 or 1 . Total energy for the shell with $n$ electrons depends on particular configuration $\left\{n_{\alpha}\right\}$

$$
E_{n_{1} \ldots n_{G}}=\sum_{\alpha=1}^{G} \epsilon_{f \alpha} n_{\alpha}+\frac{1}{2} U\left(\Sigma_{\alpha} n_{\alpha}\right)\left[\left(\Sigma_{\alpha} n_{\alpha}\right)-1\right] .
$$

Many-body wave function is also characterized by a set of numbers $\left\{n_{\alpha}\right\}$, i.e. $\left|n_{1} \ldots n_{G}\right\rangle$. Energy $E_{n_{1} \ldots n_{G}}$ remains degenerate, which can be calculated as product of how many combinations exists to accommodate electrons in each sublevel, i.e. $C_{n_{1}}^{d_{1}} \times \ldots C_{n_{\alpha}}^{d_{\alpha}} \times \ldots C_{n_{G}}^{d_{\alpha}}$. Let us further introduce probabilities $\psi_{n_{1} \ldots n_{G}}$ to find a shell in a given state with energy $E_{n_{1} \ldots n_{G}}$. Sum of all probabilities should be equal to 1, i.e.

$$
\sum_{n_{1}=0}^{d_{1}} \ldots \sum_{n_{\alpha}=0}^{d_{\alpha}} \ldots \sum_{n_{G}=0}^{d_{G}} C_{n_{1}}^{d_{1}} \ldots C_{n_{\alpha}}^{d_{\alpha}} \ldots C_{n_{G}}^{d_{G}} \psi_{n_{1} \ldots n_{G}}^{2}=1 .
$$


There are two Green functions in Gutzwiller method: impurity Green function $\hat{G}_{f}(i \omega)$ and quasiparticle Green function $\hat{G}_{g}(\omega)=\hat{b}^{-1} \hat{G}_{f}(\omega) \hat{b}^{-1}$, where matrix coefficients $\hat{b}$ represent generalized mass renormalizations parameters. All matrices are assumed to be diagonal and have diagonal elements enumerated as follows: $G_{1}(\omega), \ldots G_{\alpha}(\omega), \ldots G_{G}(\omega)$. Each element in the Green function is represented as follows

$$
\begin{gathered}
G_{g \alpha}(\omega)=\frac{1}{\omega-\lambda_{\alpha}-b_{\alpha}^{2} \Delta_{\alpha}(\omega)}, \\
G_{f \alpha}(\omega)=b_{\alpha}^{2} G_{g \alpha}(\omega) .
\end{gathered}
$$

and determines a mean number of electrons in each sublevel

$$
\bar{n}_{\alpha}=d_{\alpha} T \sum_{i \omega} G_{g \alpha}(i \omega) e^{i \omega 0^{+}}
$$

The total mean number of electrons is thus: $\bar{n}=\sum_{\alpha=1}^{G} \bar{n}_{\alpha}$. Hybridization function $\hat{\Delta}(i \omega)$ is the matrix which is assumed to be diagonal, and it has diagonal elements enumerated as follows: $\Delta_{1}(\omega), \ldots \Delta_{\alpha}(\omega), \ldots \Delta_{G}(\omega)$. Mass renormalizations $Z_{\alpha}=b_{\alpha}^{2}$ are determined in each sublevel.

Diagonal elements for the self-energy are

$$
\Sigma_{\alpha}(\omega)=\omega+\mu-\epsilon_{f \alpha}-\Delta_{\alpha}(\omega)-G_{\alpha}^{-1}(\omega)=\omega\left(1-\frac{1}{b_{\alpha}^{2}}\right)-\epsilon_{f \alpha}-\frac{\lambda_{\alpha}}{b_{\alpha}^{2}} .
$$

Here:

$$
\begin{aligned}
b_{\alpha} & =R_{\alpha} L_{\alpha} \sum_{n_{1}=0}^{d_{1}} \ldots \sum_{n_{\alpha}=1}^{d_{\alpha}} \ldots \sum_{n_{G}=0}^{d_{G}} C_{n_{1}}^{d_{1}} \ldots C_{n_{\alpha}-1}^{d_{\alpha}-1} \ldots C_{n_{G}}^{d_{G}} \psi_{n_{1} \ldots n_{\alpha} \ldots n_{G}} \psi_{n_{1} \ldots n_{\alpha}-1 \ldots n_{G}} \\
L_{\alpha} & =\left(1-\sum_{n_{1}=0}^{d_{1}} \ldots \sum_{n_{\alpha}=1}^{d_{\alpha}} \ldots \sum_{n_{G}=0}^{d_{G}} C_{n_{1}}^{d_{1}} \ldots C_{n_{\alpha}-1}^{d_{\alpha}-1} \ldots C_{n_{G}}^{d_{G}} \psi_{n_{1} \ldots n_{\alpha} \ldots n_{G}}^{2}\right)^{-1 / 2} \\
R_{\alpha} & =\left(1-\sum_{n_{1}=0}^{d_{1}} \ldots \sum_{n_{\alpha}=0}^{d_{\alpha}-1} \ldots \sum_{n_{G}=0}^{d_{G}} C_{n_{1}}^{d_{1}} \ldots C_{n_{\alpha}}^{d_{\alpha}-1} \ldots C_{n_{G}}^{d_{G}} \psi_{n_{1} \ldots n_{\alpha} \ldots n_{G}}^{2}\right)^{-1 / 2}
\end{aligned}
$$

The generalization of the non-linear equations (23) has the form

$$
\begin{aligned}
0= & {\left[E_{n_{1} \ldots n_{G}}+\Lambda-\left(\Sigma_{\alpha}^{G} \lambda_{\alpha} n_{\alpha}\right)\right] \psi_{n_{1} \ldots n_{G}}+} \\
& \sum_{\alpha=1}^{G} n_{\alpha}\left[T \Sigma_{i \omega} \Delta_{\alpha}(i \omega) G_{g \alpha}(i \omega)\right] b_{\alpha}\left[R_{\alpha} L_{\alpha} \psi_{n_{1} \ldots n_{\alpha}-1 \ldots n_{G}}+b_{\alpha} L_{\alpha}^{2} \psi_{n_{1} \ldots n_{\alpha} \ldots n_{G}}\right]+ \\
& \sum_{\alpha=1}^{G}\left(d_{\alpha}-n_{\alpha}\right)\left[T \Sigma_{i \omega} \Delta_{\alpha}(i \omega) G_{g \alpha}(i \omega)\right] b_{\alpha}\left[R_{\alpha} L_{\alpha} \psi_{n_{1} \ldots n_{\alpha}+1 \ldots n_{G}}+b_{\alpha} R_{\alpha}^{2} \psi_{n_{1} \ldots n_{\alpha} \ldots n_{G}}\right] .
\end{aligned}
$$

${ }^{1}$ For a review, see, A. Georges, G. Kotliar, W. Krauth, and M. Rozenberg, Rev. Mod. Phys. 68, 13 (1996).

2 For a review, see, e.g., Theory of the Inhomogeneous Electron Gas, edited by S. Lundqvist and S. H. March (Plenum, New York, 1983).

3 V. I. Anisimov, A. I. Poteryaev, M. A. Korotin, A. O. Anokhin, and G. Kotliar, J. Phys.: Condens. Matter 35, 7359 (1997).
4 A. Lichtenstein and M. Katsnelson, Phys. Rev. B 57, 6884 (1998).

5 G. Kotliar and S. Y. Savrasov, in New Theoretical approaches to strongly correlated systems, edited by A. M. Tsvelik, (Kluwer Academic Publishers, the Netherlands, 2001), p. 259, (available in cond-mat/020824); S. Biermann, F. Aryasetiawan, and A. Georges, Phys. Rev. Lett. 90, 086402 (2003). 
6 S. Y. Savrasov and G. Kotliar, Phys. Rev. B 69, 245101 (2004).

7 I.A. Nekrasov, K. Held, N. Blumer, A.I. Poteryaev, V.I. Anisimov, and D. Vollhardt, Eur. Phys. J. B18, 55 (2000).

8 K. Held, G. Keller, V. Eyert, D. Vollhardt, and V.I. Anisimov, Phys. Rev. Lett. 86, 5345 (2001).

9 A. I. Lichtenstein, M. I. Katsnelson, and G. Kotliar, Phys. Rev. Lett. 87, 067205 (2001).

${ }^{10}$ K. Held, A.K. McMahan, and R.T. Scalettar, Phys. Rev. Lett. 87, 276404 (2001).

11 S. Savrasov, G. Kotliar, and E. Abrahams, Nature 410, 793 (2001).

12 Xi Dai, S. Savrasov, G. Kotliar, A. Migliori, H. Ledbetter and E. Abrahams, Science 300, 953 (2003).

13 S. Y. Savrasov and G. Kotliar, Phys. Rev. Lett 90, 056401 (2003).

14 K. Held, I. A. Nekrasov, G. Keller, V. Eyert, N. Bluemer, A. K. McMahan, R. T. Scalettar, Th. Pruschke, V. I. Anisimov, and D. Vollhardt, Psi-k Newsletter \#56 (April 2003), p. 65; A. I. Lichtenstein, M. I. Katsnelson, and G. Kotliar, in Electron Correlations and Materials Properties, ed. by A. Gonis, N. Kioussis and M. Ciftan (Kluwer Academic, Plenum Publishers, 2002) p. 428.

15 P. W. Anderson, Phys. Rev. 124, 41 (1961).

${ }^{16}$ For a review, see, e.g., M. Jarrell, and J. E. Gubernatis, Physics Reports, 269, 133 (1996).
17 H. Jeschke and G. Kotliar, Rutgers University preprint, 2003.

18 S. Florens and A. Georges Phys. Rev. B 66, 165111 (2002).

19 K. Haule, S. Kirchner, J. Kroha, and P. Wölfle, Phys. Rev. B 64, 155111 (2001).

20 S. H. Vosko, L. Wilk, and M. Nusair, Can. J. Phys. 58, 1200 (1980).

${ }^{21}$ D. M. Ceperley and B. J. Alder, Phys. Rev. Lett. 45, 566 (1980).

22 M. Gutzwiller, Phys. Rev. 134, A923 (1964).

23 G. Kotliar and A. E. Ruckenstein, Phys. Rev. Lett. 57, 1362 (1986).

24 R. Fresard and G. Kotliar, Phys. Rev. B. 56, 12909 (1997).

${ }^{25}$ H. Hasegawa, Phys. Rev. B 56, 1196 (1997).

26 J. Hubbard, Proc. Roy. Soc. (London) A 281, 401 (1964).

27 R. Bulla, T. A. Costi, and D. Vollhardt, Phys. Rev. B 64, 045103 (2001).

28 S. Florens, A. Georges, G. Kotliar, and O. Parcollet, Phys. Rev. B 66, 205102 (2002).

29 See, e.g., D. D. Johnson, Phys. Rev. B 38, 12807 (1988).

30 W. Nolting, and W. Borgiel, Phys. Rev. B 39, 6962(1989).

31 L. M. Roth, Phys. Rev. 184, 451 (1969).

32 H. J. Vidberg and J. W. Serene, Journal of Low Temperature Physics, 29, 179 (1977). 